Dynamic Reconfiguration:

\section{Architectures and Algorithms}

(Series in Computer Science)

Ramachandran Vaidyanathan and Jerry L. Trahan

Plenum Publishing, 2003, \$159.00, 0-306-48189-8

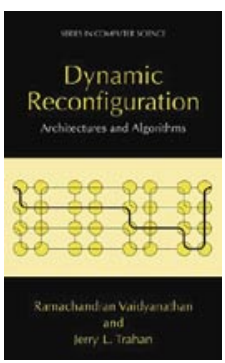

Fast computation of computer algo-

rithms is an important research area in

applied computer science. Over time,

the number of computing elements

has increased, and different ways of

connecting them have evolved. Now,

this communication medium can be

dynamically configured to achieve

fast solutions for many computing

algorithms. This book is dedicated to the study of these dynamically configured architectures.

The authors present a single computational platform called R-mesh (reconfigurable mesh), which is representative of many existing similar architectures and runs most of the algorithms described in the book.

Chapter 1 offers an introduction to dynamically reconfigurable architecture and R-mesh. It presents some simple algorithms to illustrate the use of the model.

Chapter 2 details R-mesh and the coding conventions used to describe algorithms. A collection of algorithms from arithmetic and graph theory illustrates the applications. Chapter 3 discusses the extensions and restrictions of the R-mesh model. It also describes other bus-based models, optical models, and field programmable gate arrays (FPGAs) and their relationships with R-mesh in terms of computing power and implementation.

The next four chapters are devoted to algorithms in different areas: fast arithmetical algorithms for addition, multiplication, and division; sorting algorithms and speed-efficiency concerns; graph algorithms for trees; and list ranking. Chapter 7 is devoted to computational geometry and image processing.

Chapter 8 discusses scalability problems for reconfigurable architectures, addressing the relationship between problem size and machine size. Chapter 9 goes into the computational complexity of reconfigurable architectures and presents comparisons with other models, as well as with Turing machines and circuit complexity classes. Chapter 10 addresses the use of fiber optic buses in reconfigurable architectures. Finally, chapter 12 details the runtime configuration for FPGA and describes how
R-mesh can be implemented on an FPGA platform.

The book is well written and has ample illustrations and examples. Issues are addressed in both practical and theoretical terms. The book will be useful as a text at the graduate level and may also prove valuable to the research and industrial communities.-Arun Ektare

\section{Immunity-Based Systems}

Yoshiteru Ishida

Springer-Verlag, 2004, \$89.95, ISBN: 3-540-00896-9

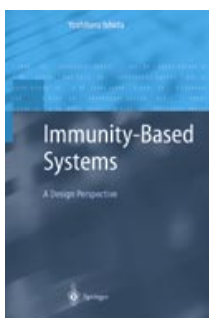

Biologically inspired systems are currently of great interest to researchers in the software engineering and AI (artificial intelligence) fields for two reasons. The first is integration. Biological systems are highly integrated dispersed subsystems that work with great collaborative synchronization. The second reason is self-

maintenance. In the age of open networks and connected enterprises, self-maintenance is high on the agenda of both software engineers and users. This book focuses on immunity systems, which demonstrate both integration and self-maintenance.

Introductory chapters cover the components of biological systems; the use of analogy and metaphor in system development, particularly in $\mathrm{AI}$; and the different computationally oriented views of biological systems. Subsequent chapters discuss the immunity-based system models. Sensor networks as self-organizing networks are also discussed, as well as the use of immunity-based systems in agents.

The book also covers the multi-agent framework and its applications; the concepts of the framework and an immune algorithm; and an application of this framework to neutralize noise.

Later chapters describe the applications of immunebased systems in a slightly different field of distributed information systems. At this stage, one might hope that security, as an important application of immune systems in developing distributed systems, would be discussed, but the author takes the agents and robots research viewpoint; hence, the focus is still on multi-agent systems.

With the increasing research in cognitive robots and biologically inspired machines, this book is a must-read for professionals in these fields.-Alladin Ayesh $Q$

Reprinted from Computing Reviews, (๖ 2004 ACM, http://www.reviews.com 